\title{
$\widehat{A}$ Madridge \\ madridge Journal of Neuroscience \\ Interconnecting Scientific World
}

Research Article

Open Access

\section{Comorbidity in Children and Adolescents with Tics and Tourette's Disorder}

\author{
Abdulhadi A Alhabbad ${ }^{1}$, Pappu S Reddy ${ }^{2 \star}$ and Saleh A Altwaijri ${ }^{2}$ \\ ${ }^{\prime}$ Division of Child and Adolescent Psychiatry, Department of Psychiatry, Queen's University, Canada \\ ${ }^{2}$ Division of Child Psychiatry, Department of Psychiatry, Queen's University, Canada
}

\section{Article Info}

\author{
*Corresponding author: \\ Pappu S Reddy \\ Associate Professor \\ Queen's University \\ Royal Victoria Hospital, Barrie \\ Ontario \\ Canada \\ Tel: +13433330047/+17057289802 \\ E-mail: reddyp@rvh.on.ca
}

Received: September 07, 2017

Accepted: October 20, 2017

Published: October 26, 2017

Citation: Alhabbad AA, Reddy PS, Altwaijri SA. Comorbidity in Children and Adolescents with Tics and Tourette's Disorder. Madridge J Neurosci. 2017; 1(1): 17-22.

doi: $10.18689 /$ mjns-1000104

Copyright: (c) 2017 The Author(s). This work is licensed under a Creative Commons Attribution 4.0 International License, which permits unrestricted use, distribution, and reproduction in any medium, provided the original work is properly cited.

Published by Madridge Publishers

\begin{abstract}
The present study analyzed tic disorders among 50 children and adolescents aged 5-18 years. Patients were sourced from a specialist service delivering child psychiatry services in Canada. Motor and vocal tics were found among the study population, with the former being predominant. Among the comorbidities assessed, Attention Deficit Hyperactivity Disorder, Generalized Anxiety Disorder and Obsessive Compulsive Disorder emerged as the most prevalent. Sleep difficulties and Intellectual Disability were also found among a large percentage of the young people having tic disorder. The coexistence of motor and phonic tics with psychiatric comorbidities observed among the study population indicates Tourette's Disorder. More organized prospective studies and analysis of the learning disabilities and familial history of the comorbidities is warranted for a better understanding of clinical presentation and etiology of tics and Tourette's Disorder.
\end{abstract}

Keywords: Motor tics; Phonic tics; ADHD; GAD; OCD; Learning disability; Hypersensitivity.

\section{Introduction}

Tic disorders represent a group of Neurodevelopmental disorders characterized by sudden and rapid stereotyped, non rhythmic motor movements and/or vocalizations [1]. Tics are more common among children and adolescents compared to adults with manifestations of varied complexities [2]. The major diagnostic classification schemes for tic disorders include the Diagnostic and Statistical Manual of Mental Disorders Fifth edition (DSM-V) [3], the International Classification of Disease and Related Health Problems, 10th revision(ICD-10) [4] and the Tourette Syndrome Classification Study Group [5]. Each of these schemes is compatible in a broader sense, although each one defines specific disorders for different neuropsychiatric disabilities such as chronic motor or vocal tic disorder (CMT, CVT), Tourette Syndrome (TS), Transient Tic Disorder (TTD) and Non-specified Tic Disorders (NSTDs) [6].

There is a high prevalence of psychiatric comorbidities in individuals with tic disorders, with Attention Deficit Hyperactivity Disorder (ADHD) and Obsessive Compulsive Disorder $(O C D)$ being the most frequent. Anxiety, self-injurious behaviour, personality disorders, depression, conduct disorders, and Oppositional Defiant Disorder are other comorbidities that are often present in patients with tic disorder [7]. Previous studies have reported significant heterogeneity in the age and sex of this patient population. There is also considerable variation in the type of onset (abrupt vs. insidious; precipitating infections and trigger events vs. none), course of the illness (chronic with some fluctuations vs. severe exacerbations with remissions), and a variable long-term prognosis, although there is often a gradual decrease in severity of symptoms after adolescence. 
Tics are frequently comorbid with other disorders in young populations [2], with up to 80 percent of subjects meeting the diagnostic criteria for another psychiatric disorder. Meanwhile, a higher familial risk among relatives of subjects with early-onset tics and OCD has repeatedly been demonstrated, suggesting that early-onset OCD might be a sub-group with a likely genetic etiology associated more often with Tourette's Disorder and other tic disorders. Studies have shown that even though the phenotypic features of tic-related OCD are similar in children, adolescents and adults, some clinical characteristics may show developmental specificity [2]. Research on psychiatric disorders in children with tics suggests that psychiatric disorders in this population share many of the same characteristics, including rate and pattern of comorbidities, with an increasing prevalence of psychiatric disorders being diagnosed in older children and adolescents.

The present study aims to investigate phenomenology, psychiatric comorbidity, and family history in referred preschool children with distressing and disabling tic symptoms. Given the limited literature on tics in preschool and school age children, especially in semirural populations in Canada, we believe that this study will contribute to the literature and help clinicians recognize and systematically assess tics in this particular population of children and adolescents. We expect that the presence of tics among clinically referred children and youth will have high rates of psychiatric comorbidity and a family history of tics. It is often the case that tics are not noticed by the patients, with other symptoms of comorbid disorders causing emotional distress and functional impairment. This association and vulnerability needs to be studied in different subgroups including children in different geographical regions. The present study will assess tic symptoms and psychiatric comorbidities among children and adolescents and determine whether there is an association between tic severity and other correlates of comorbidity.

\section{Methods}

The subjects for the current study comprised of children and adolescents recruited in a hospital based outpatient clinic in Kingston, Ontario. Fifty young people in the age group of 5-18 years presenting for psychiatric assessment who were followed up for a period of 12 months were selected. All young people had attended a outpatient clinic in a specialist child psychiatry senvice in Kingston. Data was collated from a database based on DSM-1V-TR diagnostic criteria and clinical assessments retrospectively including tic rating scales which are routinely used in the clinic (Yale-tic rating scale). Ethical approval was obtained from Queen's University ethics committee.

All the young people had been assessed with their carers or parents. The Neurodevelopmental and Mood and Anxiety clinics. All comorbid diagnoses during their initial and follow up assessments were reviewed by completing a chart review. The diagnosis, severity and type of Tics were further ascertained using Yale Tic rating scale to identify the different types of tics evident.

The prevalence of tic disorders was compared with gender, different psychiatric comorbidities, learning disabilities, treatments pursued, family history of psychiatric disorders, and the perinatal history of the subjects. The Chi-square test was applied for testing hypotheses in data analysis. The findings are discussed with reference to the defined characteristics of Tourette's Disorder.

\section{Results}

The study population comprised of 50 subjects, with $78 \%$ boys, and $32 \%$ girls. Motor tics were found to be manifest in all individuals, with phonic tics being present in $42 \%$ of the subjects, indicating that $42 \%$ of subjects expressed both tic types. Therefore, two tic groups are defined: 'motor and phonic' and 'motor and no phonic' subgroups. Sensory phenomena (premonitory urges or somatic hypersensitivities) triggering the tics traced among the study group members are presented in Table 1. Interestingly, all the subjects showed hypersensitivities and no cases of hyposensitivity were observed. Major sensory phenomena comprised tactile, visual, and auditory hypersensitivities, of which auditory hypersensitivity predominated in both patient groups (Table 1).

Table 1. Prevalence of altered sensitivities and tics in subjects.

\begin{tabular}{|l|l|l|l|l|l|l|}
\hline & \multicolumn{3}{|l|}{ Motor and phonic } & \multicolumn{2}{l|}{ Motor no phonic } & \multicolumn{2}{l|}{ Difference } \\
\hline Hypersensitivity & $\mathrm{N}$ & $\%$ & $\mathrm{~N}$ & $\%$ & $\mathrm{X}^{2}$ & $\mathrm{p}$-value \\
\hline Visual & 1 & 4.8 & 3 & 10.3 & .516 & .473 \\
\hline Tactile & 2 & 9.5 & 3 & 10.3 & .009 & .924 \\
\hline Auditory & 3 & 14.3 & 6 & 20.7 & .338 & .561 \\
\hline Hyposensitivity & & & & & & \\
\hline Visual & 0 & 0.0 & 0 & 0.0 & NA & NA \\
\hline Tactile & 0 & 0.0 & 0 & 0.0 & NA & NA \\
\hline Auditory & 0 & 0.0 & 0 & 0.0 & NA & NA \\
\hline
\end{tabular}

Hypothesis Test: There is no significant difference among patients with both motor tics and phonic tics, and the patients with motor tics alone $(p>0.5)$.

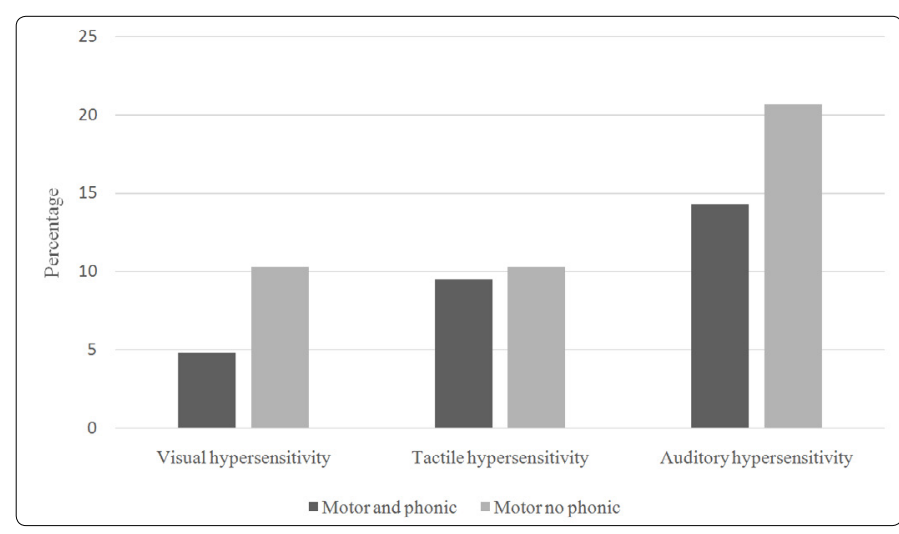

Figure 1. Prevalence of hypersensitivities in subjects based on tic type.

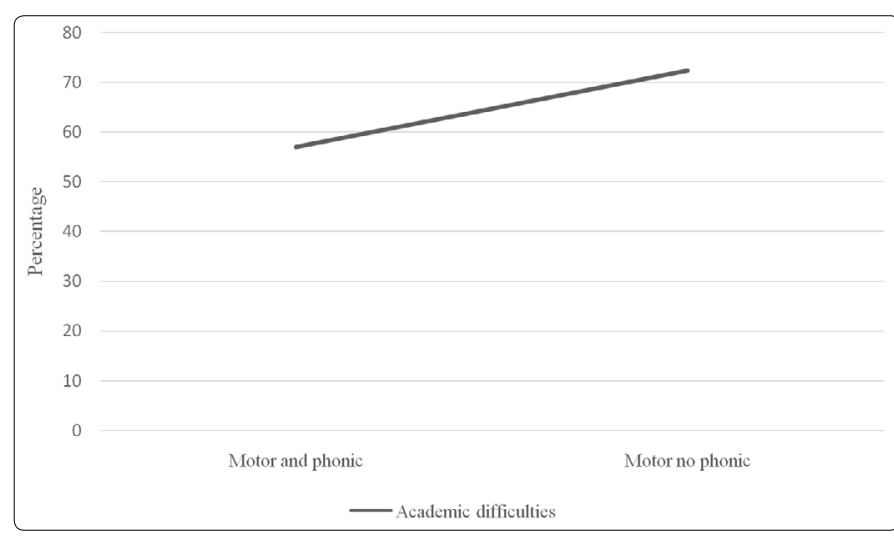

Figure 1a. Prevalence (\%) of academic difficulties in the subjects 
Psychiatric comorbidities of a wide range were found among the subjects screened for tic disorders. Table 2 illustrates the distribution of such comorbidities in the ticaffected individuals recorded during the current study. Attention Deficit Hyperactivity Disorder (ADHD) was recorded as the major comorbidity noticed across $68 \%$ of all patients, followed by Generalized Anxiety Disorder (GAD), found among $42 \%$, and Obsessive-Compulsive Disorder (OCD), found in $26 \%$ of all subjects. This observation holds true in the cases of both the motor and phonic tic patient groups individually as well. A significantly higher prevalence of learning disability was found in the motor and phonic group, a significantly higher prevalence of Oppositional Defiant Disorder (ODD) was found in the motor no phonic group. However, none of the subjects exhibited Intellectual Disorder (ID) and Psychotic Disorder (PSD). Sleep difficulty is another phenomenon frequently observed intic disorder patients. Table 3 presents the distribution of sleep disorder noticed among the patients. A total of $44 \%$ of the patients reported difficulty in sleeping, with no significant difference between the motor and phonic group (42.9\%) and the motor no phonic group (44.8\%). Most subjects also exhibited learning difficulties affecting their academic performance. Figure 1a (see Appendix 1) illustrates the prevalence of academic difficulties observed in individuals with different forms of tics. Academic difficulties were more prevalent in the motor no phonic group (72.4\%) than in the motor and phonic group (57.1\%), although this difference was not significant.

Table 2. Psychiatric comorbidities in subjects

\begin{tabular}{|l|l|l|l|l|l|l|}
\hline & \multicolumn{2}{l}{ Motor and phonic } & \multicolumn{2}{l|}{ Motor no phonic } & \multicolumn{2}{l|}{ Difference } \\
\hline Comorbidity & $\mathrm{N}$ & $\%$ & $\mathrm{~N}$ & $\%$ & $\chi^{2}$ & $\mathrm{p}$-value \\
\hline ADHD & 14 & 66.7 & 20 & 69.0 & .03 & .863 \\
\hline ASD & 1 & 4.8 & 2 & 6.9 & .098 & .754 \\
\hline Learning disability & 5 & 23.8 & 0 & 0.0 & 7.672 & $.006^{\star}$ \\
\hline GAD & 8 & 38.1 & 13 & 44.8 & .227 & .634 \\
\hline Social phobia & 1 & 4.8 & 3 & 10.3 & .516 & .473 \\
\hline OCD & 8 & 38.1 & 5 & 17.2 & 2.753 & .097 \\
\hline Panic disorder & 1 & 4.8 & 1 & 3.4 & .055 & .815 \\
\hline SAD & 1 & 4.8 & 1 & 3.4 & .055 & .815 \\
\hline Specific phobia & 0 & 0.0 & 1 & 3.4 & .739 & .39 \\
\hline Depression & 2 & 9.5 & 1 & 3.4 & .797 & .372 \\
\hline Bipolar disorder & 1 & 4.8 & 0 & 0.0 & 1.409 & .235 \\
\hline Intellectual disability & 0 & 0.0 & 0 & 0.0 & NA & NA \\
\hline ODD & 0 & 0.0 & 5 & 17.2 & 4.176 & $.041^{*}$ \\
\hline Enuresis & 0 & 0.0 & 3 & 10.3 & 2.311 & .128 \\
\hline Encopresis & 0 & 0.0 & 1 & 3.4 & .739 & .39 \\
Psychotic disorder & 0 & 0.0 & 0.0 & 0.0 & NA & NA \\
\hline
\end{tabular}

${ }^{*} p$-value is significant at the .05 level

Note. $A D H D=$ attention deficit hyperactivity disorder; $A S D=$ autistic spectrum disorder; $G A D=$ generalized anxiety disorder; $O C D=$ obsessive compulsive disorder; $\mathrm{SAD}=$ separation anxiety disorder; $\mathrm{ODD}=$ oppositional defiant disorder.

Hypothesis Test: There is a significant difference among patients with both motor tics and phonic tics, and the patients with motor tics alone for learning disability and ODD $(p<.05)$.

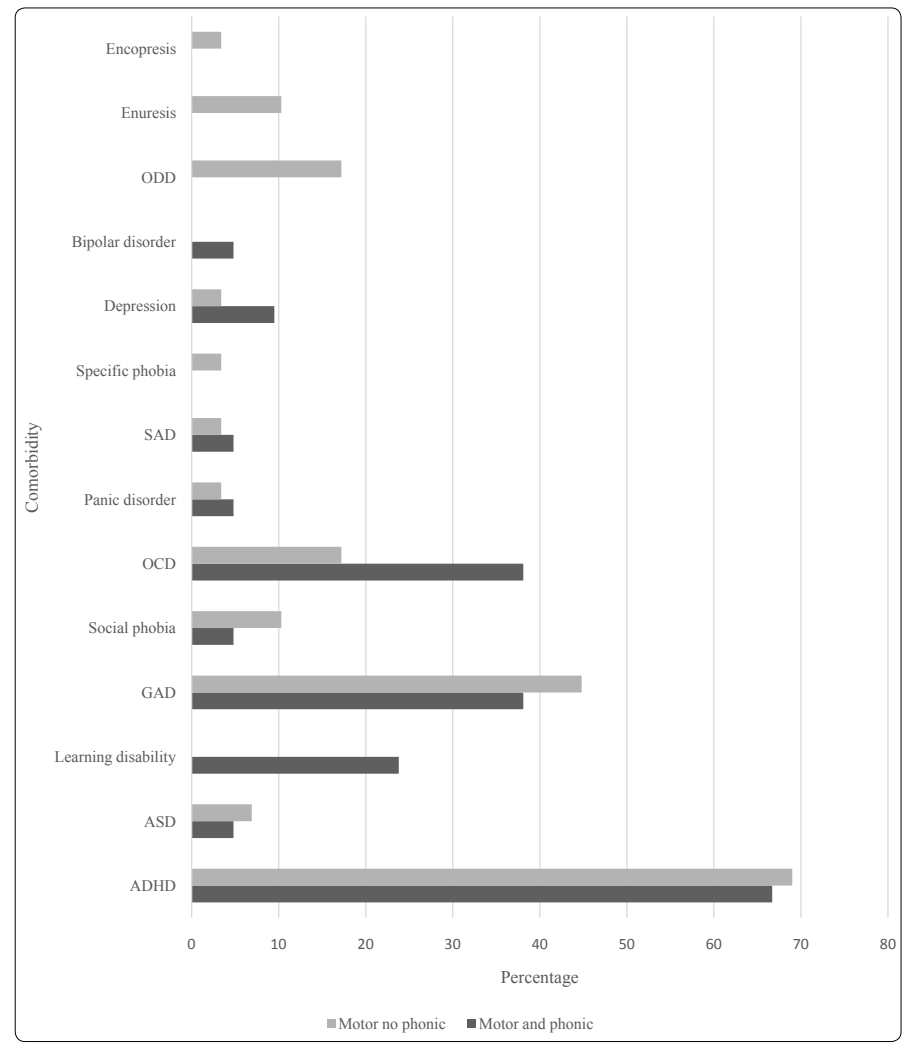

Figure 2. Psychiatric comorbidities in subjects based on tic type.

Note. $A D H D=$ attention deficit hyperactivity disorder; $A S D$ = autistic spectrum disorder; $\mathrm{GAD}$ = generalized anxiety disorder; $\mathrm{OCD}=$ obsessive compulsive disorder; $\mathrm{SAD}=$ separation anxiety disorder; ODD = oppositional defiant disorder.

Investigations into the medication and treatment options for tic disorder patients have revealed psychotherapy as well as other medications such as antidepressants, antipsychotics, and stimulants being used. Data on psychotherapy is provided in Table 3. The survey on treatment indicated $42.9 \%$ of the subjects opted for psychotherapy, with a similar prevalence across the two groups. Details of the medications received by the subjects are presented in Table 4 . Of the 50 subjects, 49 opted for medication. Stimulants are the category of drugs received by the majority of the subjects (44\%), followed by antipsychotics (20\%), and antidepressants (18\%). None of the patients received more than 3 types of medications (Table 4). Duration of treatment ranged from $<1$ year to more than 5 years. However, only $2 \%$ of the treated subjects were found undergoing follow-up treatment for more than 5 years. A significantly larger percentage of patients in the motor and phonic group were taking medication for $<1$ year $(52.4 \%$ vs. $24.1 \%$, while a significantly larger percentage of patients in the motor no phonic group were taking medication for 1 to 5 years $(20.7 \%)$, with no individual in the motor and phonic group falling into this category.

Table 3. Psychotherapy and sleep difficulty in the subjects (\%)

\begin{tabular}{|l|l|l|l|l|l|l|}
\hline & \multicolumn{3}{|l|}{ Motor and phonic } & \multicolumn{2}{l|}{ Motor no phonic } & \multicolumn{2}{l|}{ Difference } \\
\hline Variable & $\mathrm{N}$ & $\%$ & $\mathrm{~N}$ & $\%$ & $\mathrm{X}^{2}$ & $\mathrm{p}$-value \\
\hline Psychotherapy & 9 & 42.9 & 12 & 41.4 & .063 & .801 \\
\hline Sleep difficulty & 9 & 42.9 & 13 & 44.8 & .019 & .89 \\
\hline
\end{tabular}

Hypothesis Test: There is no significant difference among patients with both motor tics and phonic tics, and the patients with motor tics alone $(p>0.5)$. 


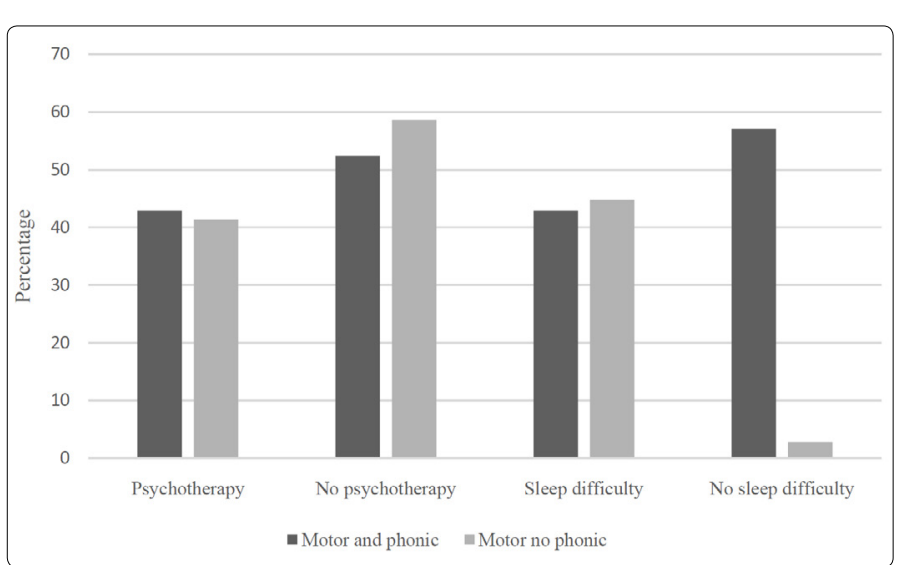

Figure 3. Psychotherapy and sleep difficulty in subjects based on tic type.

Table 4. Current medication status of subjects as per the symptomology (\%)

\begin{tabular}{|l|l|l|l|l|l|l|}
\hline & \multicolumn{2}{|l|}{ Motor and phonic } & \multicolumn{2}{l|}{ Motor no phonic } & \multicolumn{2}{l|}{ Difference } \\
\hline Variable & $\mathrm{N}$ & $\%$ & $\mathrm{~N}$ & $\%$ & $\mathrm{X}^{2}$ & $\mathrm{p}$-value \\
\hline Takes medication & 13 & 61.9 & 19 & 65.5 & .188 & .665 \\
\hline Medication type & & & & & & \\
\hline Antidepressants & 6 & 28.6 & 3 & 10.3 & 2.741 & .098 \\
\hline Antipsychotics & 4 & 19.0 & 6 & 20.7 & .021 & .886 \\
\hline Stimulants & 6 & 28.6 & 16 & 55.2 & 3.498 & .061 \\
\hline$>3$ types & 0 & 0.0 & 0 & 0.0 & 3.743 & .053 \\
\hline Duration of current medication (years) \\
\hline$<1$ & 11 & 52.4 & 7 & 24.1 & 4.48 & $.034^{\star}$ \\
\hline $1-5$ & 0 & 0.0 & 6 & 20.7 & 5.053 & $.025^{\star}$ \\
\hline$>5$ & 1 & 4.8 & 0 & 0.0 & 1.509 & .219 \\
\hline
\end{tabular}

Hypothesis Test: There is no significant difference among patients with both motor tics and phonic tics, and the patients with motor tics alone for presence and type of medication $(p>.05)$. There is a significant difference between the two groups on whether they have taken medication for less than 1 year, or $1-5$ years $(p<0.5)$.

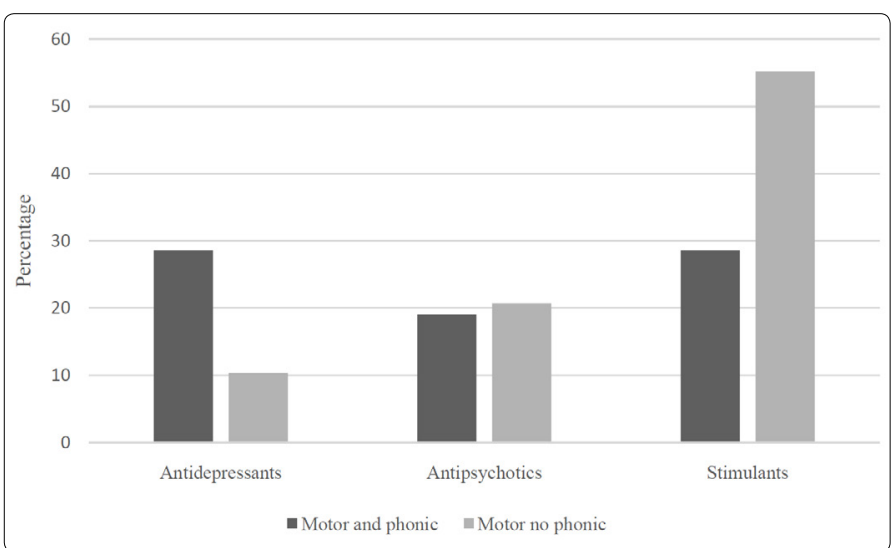

Figure 4. Medication taken by tic type.

The perinatal history of the tic disorder patients is presented in Table 5. None of the mothers of tic subjects had gestational diabetes or received any medication and vaccines before the birth of these children. Most subjects were born by normal delivery at term (74\%). Spontaneous normal pre-term birth was the second dominant mode $(8 \%)$, followed by induced normal, and induced C-section (6\% each). There were no significant differences between the two groups on these measures.
Table 5. Perinatal history of the subjects

\begin{tabular}{|c|c|c|c|c|c|c|}
\hline \multirow[b]{2}{*}{ During Pregnancy } & \multicolumn{6}{|c|}{ Motor and phonic/Motor no phonic Difference } \\
\hline & $N$ & $\%$ & $\mathrm{~N}$ & $\%$ & $x^{2}$ & p-value \\
\hline Medical problem & 0 & 0.0 & 0 & 0.0 & NA & NA \\
\hline Gestational diabetes & 0 & 0.0 & 0 & 0.0 & NA & NA \\
\hline Medication & 0 & 0.0 & 0 & 0.0 & NA & NA \\
\hline Vaccines & 0 & 0.0 & 0 & 0.0 & NA & NA \\
\hline \multicolumn{7}{|l|}{ Delivery } \\
\hline \multicolumn{7}{|c|}{ Normal spontaneous vertex } \\
\hline -At term & 16 & 76.2 & 21 & 72.4 & .09 & .764 \\
\hline -Pre-term & 1 & 4.8 & 3 & 10.3 & .516 & .473 \\
\hline \multicolumn{7}{|l|}{ Induction labor } \\
\hline -At term & 0 & 0.0 & 3 & 10.3 & 2.877 & .09 \\
\hline -Pre-term & 2 & 9.5 & 0 & 0.0 & 2.311 & .128 \\
\hline \multicolumn{7}{|l|}{ C-section } \\
\hline -At term & 1 & 4.8 & 2 & 6.9 & .098 & .754 \\
\hline -Pre-term & 0 & 0.0 & 0 & 0.0 & NA & NA \\
\hline
\end{tabular}

Hypothesis Test: There is no significant difference among patients with both motor tics and phonic tics, and the patients with motor tics alone ( $p>0.05)$.

The history of various psychiatric disorders traced in the families of the tic subjects is presented in Table 6 . Among the 20 different types of psychiatric disorders verified in the family history of the tic affected individuals, depression emerged as the prevalent disorder existing in $26 \%$ of the families, followed by tic disorder (24\%). Other major forms of disorders included autistic spectrum disorder, schizophrenia, ADHD, and OCD. Panic disorder $(P D)$, intellectual disorder (ID), separation anxiety disorder (SAD), specific phobia (SPP), oppositional defiant disorder (ODD), encopresis (ENC) and PSD were not traced in any of the families.

Table 6. Family history of the subjects

\begin{tabular}{|c|c|c|c|c|c|c|}
\hline \multirow[b]{2}{*}{ Disorder } & \multicolumn{2}{|c|}{ Motor and phonic } & \multicolumn{2}{|c|}{ Motor no phonic } & \multicolumn{2}{|c|}{ Difference } \\
\hline & $\mathrm{N}$ & $\%$ & $N$ & $\%$ & $x^{2}$ & $\mathrm{p}$-value \\
\hline ADHD & 3 & 14.3 & 5 & 17.2 & .141 & .707 \\
\hline ASD & 6 & 28.6 & 3 & 10.3 & 2.448 & .118 \\
\hline Learning disability & 2 & 9.5 & 1 & 3.4 & .702 & .402 \\
\hline GAD & 6 & 28.6 & 7 & 24.1 & .053 & .818 \\
\hline Social phobia & 1 & 4.8 & 1 & 3.4 & .036 & .849 \\
\hline OCD & 2 & 9.5 & 6 & 20.7 & 1.436 & .246 \\
\hline Panic disorder & 0 & 0.0 & 0 & 0.0 & NA & NA \\
\hline SAD & 0 & 0.0 & 0 & 0.0 & NA & NA \\
\hline Specific phobia & 0 & 0.0 & 0 & 0.0 & NA & NA \\
\hline Depression & 7 & 33.3 & 6 & 20.7 & .793 & .373 \\
\hline Bipolar disorder & 3 & 14.3 & 0 & 0.0 & 4.172 & $.041^{*}$ \\
\hline Intellectual disability & 0 & 0.0 & 0 & 0.0 & NA & NA \\
\hline ODD & 0 & 0.0 & 0 & 0.0 & NA & NA \\
\hline Enuresis & 0 & 0.0 & 1 & 3.4 & .786 & .375 \\
\hline Encopresis & 0 & 0.0 & 0 & 0.0 & NA & NA \\
\hline Schizophrenia & 4 & 19 & 5 & 17.2 & .004 & .948 \\
\hline Substance abuse & 3 & 14.3 & 3 & 10.3 & .087 & .769 \\
\hline Tourette's syndrome & 0 & 0.0 & 1 & 3.4 & .786 & .375 \\
\hline Other tic disorder & 6 & 28.6 & 6 & 20.7 & .281 & .596 \\
\hline Other psychotic disorde & 0 & 0.0 & 0 & 0.0 & NA & NA \\
\hline
\end{tabular}

${ }^{*} \mathrm{p}$-value is significant at the .05 level

Note. $A D H D=$ attention deficit hyperactivity disorder; $A S D=$ autistic spectrum disorder; $G A D=$ generalized anxiety disorder; $O C D=$ obsessive compulsive disorder; $\mathrm{SAD}=$ separation anxiety disorder; $\mathrm{ODD}=$ oppositional defiant disorder.

Hypothesis Test: There is a significant difference among patients with both motor tics and phonic tics, and the patients with motor tics alone for family history of bipolar disorder $(p<0.05)$. 


\section{Discussion}

Tourette's Syndrome (TS) refers to a neurodevelopmental disorder with complex manifestations of neurological and psychiatric symptoms. TS was renamed Tourette's Disorder in the DSM-V, and the tics defined as "sudden, rapid, recurrent, nonrhythmic motor movements or vocalizations, generally preceded by urge" [8]. As per DSM-V, a Tourette's Disorder can only be classified as such when the individual presents "multiple motor and vocal tics present at some time with the disorder beginning before the age of 18 years and lasting more than one year, and where the tics are not secondary to a physiological substance or other neurological disorder"[9]. Studies on the prevalence of Tourette's Disorder from different international communities have reported affliction of around $1 \%$ of school children, with boys being at a higher risk (2.4 times) $[10,11]$. Outcomes of the present study also showed a similar trend with $78 \%$ of the subjects being male. Prenatal androgen related masculinization and thinning of the frontoparietal cortex have been attributed for the relative higher risk of boys over girls to this disorder [2].

The current study has recorded the presence of motor and phonic tics, preceded by somatic hypersensitivity among the subjects. Auditory hypersensitivities were the most common in both patient groups. Indeed, an association between hypersensitivity and tics has been reported in Tourette's Disorder. This has been described as a crucial phenomenon often linked with other aspects of the syndrome as well [12]. Also, as many as $50 \%$ of patients report their tic symptomatology disappearing in their adult life [9].

Most children affected by Tourette's Disorder often exhibit comorbidities, especially of a psychiatric nature. Population studies on the association of Tourette's Disorder with psychiatric comorbidities at the clinical and community level have reported only $10 \%$ of the cases as free from the latte [13]. The current investigation recorded more than 10 different psychiatric comorbidities among the subjects, with $A D H D, G A D$ and $O C D$ being the most prevalent. Extensive studies on the comorbidities of Tourette's Disorder have reported $A D H D$ and $O C D$ as the predominant ones $[14,15,16$, $17,18,19,20]$ Most of the psychiatric disorders did not differ significantly the motor and phonic group and the motor no phonic group, while learning disability was more prevalent in the former and ODD was more prevalent in the latter.

Sleep disorders were recorded among $44 \%$ of the subjects in the present study. A similar prevalence of this phenomenon was found in a systematic review by Ferreira et al [21] with insomnia, sleepwalking, and hypersomnia being identified as the major forms of sleep disorders [22]. Sleep tribulations associated with Tourette's Disorder and tic disorders have been shown to influence the frequency and severity of tics [23].

In addition, the current study has identified learning difficulties as another major challenge among the subjects, with a prevalence of $71.4 \%$. However, earlier studies have reported a rather lower prevalence of this comorbidity in patients with Tourette's Disorder [21]. The higher rate of occurrence of learning disability in the current study suggests that further investigation is needed to understand its impact on the severity of the disorder, and the influence of genetics, social factors, and other parameters.

The treatment regimens undertaken by the subjects in the present study comprised medications and psychotherapy. Stimulants were the predominant drugs received by the subjects, and more than $40 \%$ of the individuals attended psychotherapy sessions. While significantly more patients in the motor no phonic group were taking medication for 1 to 5 years, this may be the result of an indirect effect of length of time since diagnosis, which is data that were not available to us. It does warrant further investigation, as it may be indicative of individuals in this group being more dependent on medication in the long-term.

The family history of psychiatric disorders tracked for the subjects has indicated depression as the most prevalent disorder observed among family members. The second most common was tic disorders. Moreover, many psychiatric disorders expressed by the tic subjects have been traced in the subjects' families. However, a more structured investigation on tracking the occurrence of these disorders in the subjects, their immediate family members, earlier generations, and so forth, needs to be carried out to understand the persistence of any disorders from childhood to adulthood, or the transition of one form of disorder to another along with advancing age. Little is known about the etiology of Tourette's Disorder; however, the involvement of genetic and neuro-immunological factors is suggested in the current literature [24]. Corticostriato-thalamo-cortical circuits of the brain become affected leading to the disorientation of motor, cognitive, and motivational parts of behaviour [25].

\section{Conclusion}

The current study indicated the association of psychiatric comorbidities like ADHD, GAD, and OCD at a high frequency, and some other psychiatric comorbidities at a lower frequency, in young people with Tourette's Disorder and tics. No significant difference was found between comorbidity for tics and Tourette's when taken separately. A high frequency of learning disabilities, and overlapping of the comorbidities in family histories of the subjects observed during the current study has provided scope for the further investigation of these aspects, which may help in enhancing the understanding of Tourette's Disorder and its management.

\section{Conflict of interest}

The authors confirm that there is no conflict of interest regarding this manuscript.

\section{Acknowledgments}

This project was funded by a grant from Queen's University. 


\section{References}

1. Abell S, Ey J. Tics in childhood. Clin Pediatr (Phila). 2009; 48: 790-791.

2. Rodgers $S$, Müller $M$, Kawohl W, et al. Sex-related and non-sex-related comorbidity subtypes of tic disorders: A latent class approach. Eur $J$ Neurol. 2014; 21(5): 700-707. doi: 10.1111/ene.12274

3. American Psychiatric Association. Diagnostic and statistical manual of mental disorders (4th ed., text rev.), 2000.

4. World Health Organization. International statistical classification of diseases and related health problems (10th ed.). Geneva WHO; 1992.

5. The Tourette Syndrome Classification Study Group. Definitions and classification of tic disorders. Arch Neurol. 1993; 50: 1013-1016.

6. Cubo E. Review of prevalence studies of tic disorders: methodological caveats. Tremor Other Hyperkinet Mov (N Y), 2012.

7. Robertson MM. The prevalence and epidemiology of Gilles de la Tourette syndrome. Part 2: tentative explanations for differing prevalence figures in GTS, including the possible effects of psychopathology, aetiology, cultural differences, and differing phenotypes. J Psychosom Res. 2008; 65: 473486. doi: 10.1016/j.jpsychores.2008.03.007

8. American Psychiatric Association. Diagnostic and Statistical Manual of Mental Disorders (Fifth ed.). Arlington, VA: American Psychiatric Publishing. pp. 5-25. 2013.

9. Hallett M. Tourette Syndrome: Update. Brain Dev. 2015; 37(7): 651-655. doi:10.1016/j.braindev.2014.11.005

10. Robertson MM. The prevalenceand epidemiology of Gilles de la Tourette Syndrome. Part 1. the epidemiological and prevalence studies. Psychosomatic Medicine. 2008; 65: 461-472.

11. Schalill L, Bitsko RH, Blumberg SJ. Prevalence of diagnosed Tourette Syndrome $\mathrm{n}$ persons aged 6-17years - United States. Morbidity and Mortality Weekly Report. 2009; 58: 581-585.

12. Cohen S, Leckman JF, Bloch MH. Clinical assessment of Tourette syndrome and tic disorders. Neurosci Biobehav Rev. 2013; 37(6): 997-1007. doi: 10.1016/j.neubiorev.2012.11.013

13. Cavanna AE, Seri S. Tourette's syndrome. Bmj. 2013; 347(aug2013): f4964-f4964. doi:10.1136/bmj.f4964
14. Cavanna AE, Servo S, Monaco F, Robertson MM. The behavioral spectrum of Gilles de la Tourette syndrome. J Neuropsychiatry Clin Neurosci. 2009; 21: 13-23. doi: 10.1176/jnp.2009.21.1.13

15. Du JC, Chiu TF, Lee KM, Wu HL, Yang YC, Hsu SY, et al. Tourette syndrome in children: an updated review. Pediatr Neonatol. 2010; 51: 255-64. doi: 10.1016/S1875-9572(10)60050-2

16. Cavanna AE, Robertson MM, Critchley HD. Schizotypal personality traits in Gilles de la Tourette syndrome. Acta Neurol Scand. 2007; 116: 385-91. doi: 10.1111/j.1600-0404.2007.00879.x

17. Coffey BJ, Biederman J, Smoller JW, Geller DA, Sarin P, Schwartz S, et al. Anxiety disorders and tic severity in juveniles with Tourette's disorder. $J$ Am Acad Child Adolesc Psychiatry. 2000; 39: 562-8. doi: 10.1097/00004583200005000-00009

18. Comings DE. Clinical and molecular genetics of ADHD and Tourette syndrome. Two related polygenic disorders. Ann N Y Acad Sci. 2001; 931: 50-83.

19. Fernández-Alvarez E. [Comorbid disorders associated with tics]. Rev Neurol. 2002; 34 Suppl 1: S122-9.

20. Kano $Y$, Ohta $M$, Nagai $Y$, Scahill L. Association between Tourette syndrome and comorbidities in Japan. Brain Dev. 2010; 32: 201-7. Epub 2009 Feb 28. doi: 10.1016/j.braindev.2009.01.005

21. Ferreira BR, Pio-Abreu JL, Januário C. Tourette's syndrome and associated disorders: a systematic review. Trends Psychiatry Psychother. 2014; 36(3): 123-133.

22. Taylor E. Sleep and tics: problems associated with ADHD. J Am Acad Child Adolesc Psychiatry. 2009; 48: 877-8. doi: 10.1097/CHI.0b013e3181af825a

23. Swain JE, Leckman JF. Tourette syndrome and tic disorders: overview and practical guide to diagnosis and treatment. Psychiatry (Edgmont). 2005; 2(7): 26-36.

24. Stern J. Tourette Syndrome. Paediatr Child Health (Oxford). 2014; 24(10): 447-451. doi: 10.1016/j.paed.2014.03.003

25. Serajee FJ, Mahbubul Huq AHM. Advances in Tourette Syndrome. Pediatr Clin North Am. 2015; 62(3): 687-701. doi: 10.1016/j.pcl.2015.03.007 